\title{
WAVENUMBER IMAGING FOR DAMAGE DETECTION AND MEASUREMENT
}

\author{
M. D. Rogge ${ }^{1}$ and P. H. Johnston ${ }^{1}$ \\ ${ }^{1}$ NASA Langley Research Center, Hampton, VA 23681
}

\begin{abstract}
This paper presents a method for analyzing ultrasonic wavefield data using the Continuous Wavelet Transform (CWT) applied in the spatial domain. Unlike data obtained by sparse arrays of transducers, full wavefield data contains information local to the structure and can be used to obtain more detailed measurements of damage type, location, size, etc. By calculating the CWT of the wavefield in the spatial domain, the wavenumber spectrum is determined for the inspected locations. Because wavenumber is affected by the local geometry and material properties of the structure through which Lamb waves propagate, the wavenumber spectrum can be analyzed to assess the location, severity, and size of damage. The technique is first applied to experimental wavefield data obtained using a laser Doppler vibrometer and automated positioning stage. The out-of-plane velocity along the length of a composite stringer was measured to detect the presence of delaminations within the composite overwrap. Next, simulated corrosion is detected and measured within an aluminum plate using the two dimensional CWT. The experimental results show the usefulness of the technique for vehicle structure inspection applications.
\end{abstract}

Keywords: Structural Health Monitoring, Wavelet Transforms, Acoustic Wavefield Imaging, Guided Waves

PACS: 43.35.Zc, 43.35.Cg, 43.60.Gk, 42.79.Qx

\section{INTRODUCTION}

Guided waves, such as Lamb waves, have found many applications towards damage detection, localization, and measurement. Permanently mounted transducers can be used to generate and detect guided waves in structures [1]. However, the number and locations of the bonded transducers limit resolution and fidelity of defect detection and measurement [2]. It is possible to supplement structural health monitoring provided by permanently mounted transducers with non-contact wavefield measurements performed over the surface of the structure $[3,4]$. Researchers have investigated multiple methods of interpreting the very large datasets obtained in this manner [5-7]. Of particular interest is 
the use of laser Doppler velocimeters to obtain the wavefield data due to its non-contact nature and its ability to scan large areas [8]. This paper presents a method for local analysis of wavefield data that effectively highlights the presence of defects and provides a means to quantify defect severity.

\section{EXPERIMENTAL METHODS}

\section{PRSEUS Composite Stringer}

Two samples were utilized in this work. First, a composite stringer consisting of a $9.5 \mathrm{~mm}$ diameter pre-cured, pultruded carbon fiber rod with carbon fiber reinforced epoxy overwrap was investigated. The stringer is part of the PRSEUS (Pultruded Rod Stitched Efficient Unified Structure) composite structural system with an overwrap layup of [+45,45,0,90,0,-45,45]. A $1.0 \mathrm{MHz}$ Panametrics V103 transducer was coupled to the end of the rod using gel couplant. The transducer was excited by a 5 -cycle $250 \mathrm{kHz}$ sine burst with amplitude $70 \mathrm{~V}$ to generate guided waves that travel along the length of the stringer. The acoustic wavefield was measured with a Polytec OFV-505 laser Doppler vibrometer (LDV) and recorded by a LeCroy 6030 8-bit oscilloscope at a rate of $50 \mathrm{MHz}$. The stringer was placed in an automated scanner and measurements were recorded every $0.5 \mathrm{~mm}$ along the length of the stringer in a linear manner at $45^{\circ}, 90^{\circ}$, and $135^{\circ}$ angular locations. The stringer was also inspected by an immersion ultrasonic probe with the sample placed on a turntable. $240 \mathrm{~mm}$ of the stringer length was inspected in $1.27 \mathrm{~mm}$ increments and $180^{\circ}$ rotation in $1^{\circ}$ increments using a $5 \mathrm{MHz}$ probe with $101.6 \mathrm{~mm}$ focal length.

The stringer contained delaminations that were unintentionally created during manufacturing. Data recorded by the LDV and X-Ray Computed Tomography (CT) crosssection images of the delamination are shown in Figure 1. The wave is seen to propagate without disruption along the stringer until it reaches $120 \mathrm{~mm}$ where scattering and mode conversion occurs, coinciding with the start of the delamination.
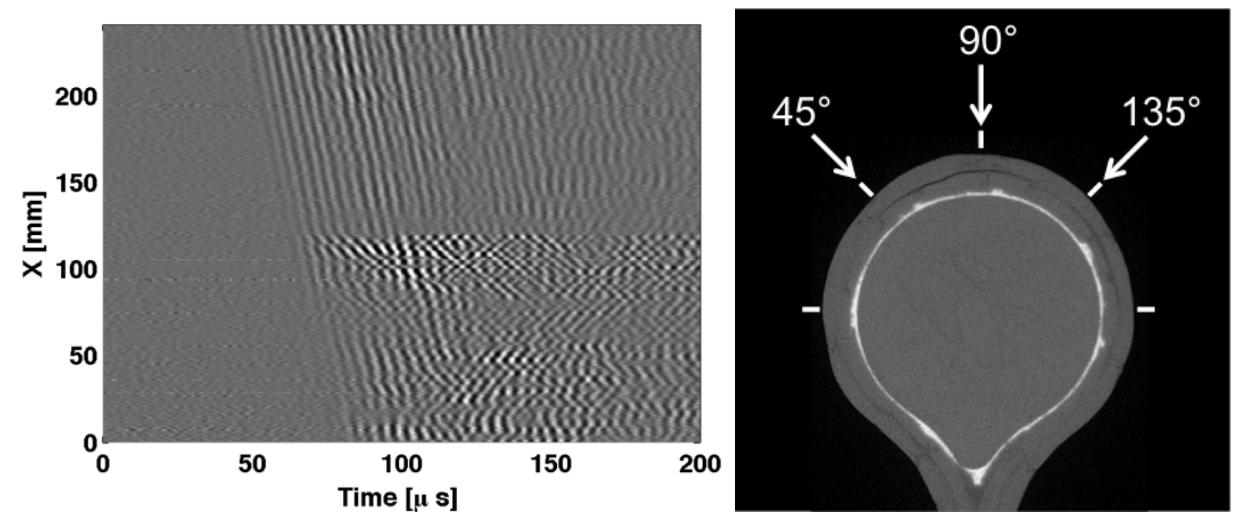

FIGURE 1. Recorded data obtained along the length of the stringer at $90^{\circ}$ angular location and an X-ray CT image of stringer cross section showing the three inspection angular locations 
The second sample is a $2.2 \mathrm{~mm}$ thick 6061 aluminum plate with four machined pockets. The pockets are designated A-D and have geometries as listed in Table 1. Both A0 and S0 mode Lamb waves were generated in this sample by a $9.6 \mathrm{~mm}$ diameter, 0.8 mm thick PZT disk bonded to the center of the plate. The transducer was excited by a 5 cycle $250 \mathrm{kHz}$ sine burst. An automated scanner performed positioning of the plate in front of the LDV. Signals were recorded on a Cartesian grid with $0.8 \mathrm{~mm}$ resolution in $100 \times 100$ $\mathrm{mm}$ square regions surrounding each defect.

Data recorded in the vicinity of defect $A$ at various times is shown in Figure 2. The incident S0 mode reaches the defect at approximately $45 \mu$ s and causes scattering of an A0 mode within and away from the defect. The A0 mode reaches the defect at approximately $65 \mu \mathrm{s}$. The circular wave front is disrupted due to slowing of the wave within the defect and A0 mode scattering is also present.

TABLE 1. Defect geometries present in the aluminum plate.

\begin{tabular}{|c|c|l|l|}
\hline Designation & Width $(\mathrm{mm})$ & Height & Depth \\
\hline A & 25 & 25 & 0.36 \\
\hline B & 25 & 25 & 0.18 \\
\hline C & 12.5 & 12.5 & 0.36 \\
\hline D & 12.5 & 12.5 & 0.18 \\
\hline
\end{tabular}
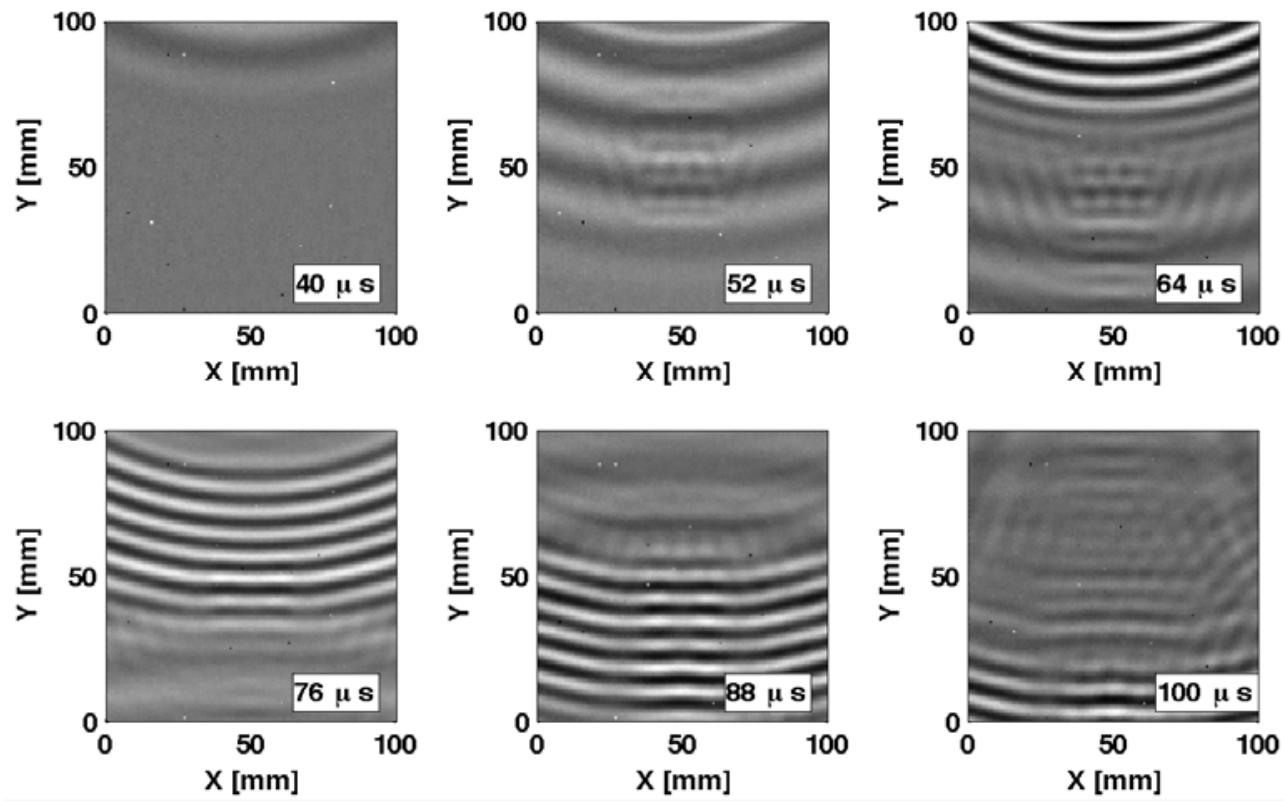

FIGURE 2. Recorded data obtained at various times in the vicinity of a $25 \times 25 \times 0.36 \mathrm{~mm}$ pocket. The S0 mode is seen interacting with the pocket at 52-64 $\mu$ s. The A0 mode is seen interacting with the pocket at 76$100 \mu \mathrm{s}$. Scattering from the edge of the plate enters the image at $100 \mu \mathrm{s}$. 
WAVENUMBER ANALYSIS METHODS

\section{$\underline{\text { 1-D Continuous Wavelet Transform }}$}

The Continuous Wavelet Transform has been used to analyze the time-frequency content of guided waves [9]. The one dimensional CWT coefficients, $C(a, b)$, of a signal $s(t)$ are given as:

$$
C(a, b)=\frac{1}{\sqrt{a}} \int_{-\infty}^{\infty} s(t) \psi^{*}\left(\frac{t-b}{a}\right) d t
$$

where $a$ is the scale, $b$ is a translational value, and $\psi(t)$ is the mother wavelet [10]. In this work, the complex Morlet mother wavelet is used and is defined as

$$
\psi(t)=\frac{1}{\sqrt{2 \pi}} e^{-t^{2} / 2} e^{i 5 t}
$$

The scale and translational value can be related to a pseudo frequency and time, respectively, and can thus be used to create a time-frequency representation of the signal where the magnitude of a coefficient is proportional to the energy at a given time and frequency. Alternatively, if the signal is a function of a spatial quantity, the CWT results in a space-wavenumber representation $[11,12]$. The use of the 1D CWT is applicable for data obtained along a linear path such as the case for the composite stringer. However, the resulting spectrum will only contain the component of the wavenumber in the direction of the linear path and therefore cannot be used for quantitative analysis in all cases.

\section{$\underline{\text { 2-D Continuous Wavelet Transform }}$}

Two-dimensional data can be processed using the 2D CWT to produce coefficients that are functions of the two spatial locations, $x$ and $y$, wavenumber, and wave propagation direction, $\theta$.

$$
C\left(a, b_{1}, b_{2}, \theta\right)=\frac{1}{\sqrt{a}} \int_{-\infty}^{\infty} \int_{-\infty}^{\infty} s(x, y) \psi^{*}\left(\frac{x-b_{1}}{a}, \frac{y-b_{2}}{a}, \theta\right) d x d y
$$

The two dimensional Morlet wavelet is defined as a complex plane wave windowed by a Gaussian function:

$$
\psi(x, y, \theta)=\frac{1}{2 \pi} e^{-\left(x^{2}+y^{2}\right) / 2} e^{i 5(x \cos \theta+y \sin \theta)}
$$

In order to obtain the wavenumber spectrum irrespective of direction, the CWT coefficients obtained for multiple directions are recorded and their magnitudes summed.

\section{RESULTS}

Figure 3 shows the data obtained along the length of the composite stringer. The incident wave is clearly seen to be propagating from the right side of the figures towards 
the left with a relatively long wavelength. The space-wavenumber representation shows the wavenumber is approximately $\mathrm{k}=30 \mathrm{~m}^{-1}$. When the wave reaches the delamination within the overwrap at approximately $\mathrm{x}=120 \mathrm{~mm}$, the wavenumber abruptly changes with energy present from $\mathrm{k}=100$ to $200 \mathrm{~m}^{-1}$. This is due to the geometry of the thin delaminated region supporting a shorter wavelength at the wave frequency. As the wave continues to propagate along the stringer, the wavenumber distribution is broadened and energy is present at $\mathrm{k}=45 \mathrm{~m}^{-1}$. The change in wavenumber demonstrates the existence of a defect at that spatial location. However, a model is necessary to identify delamination geometry based on the measured wavenumber.

Previously, multidimensional Fourier transforms have been used to identify modal content in guided waves [6]. A two-dimensional transform of the recorded data for the stringer was performed and is presented in Figure $4 \mathrm{a}$ in normalized, linear units. For comparison, the spatial spectrogram was calculated for $40-150 \mu$ s and summed as shown in Figure $4 \mathrm{~b}$. The two dimensional transform shows a bandwidth of $210-260 \mathrm{kHz}$ and 220-220 $\mathrm{m}^{-1}$. While the 2D FT is not readily interpreted, some features can be identified. The incident wave is indicated by the dark region present over the frequency bandwidth from 20-35 m-1. Another dark region is apparent from $\pm 120-210 \mathrm{~m}^{-1}$, most likely corresponding to the large response at the delamination at $x=120 \mathrm{~mm}$. The good agreement between the two transforms suggests the spatial spectrogram can be used to measure wavenumber spectra as a function of location within a structure.
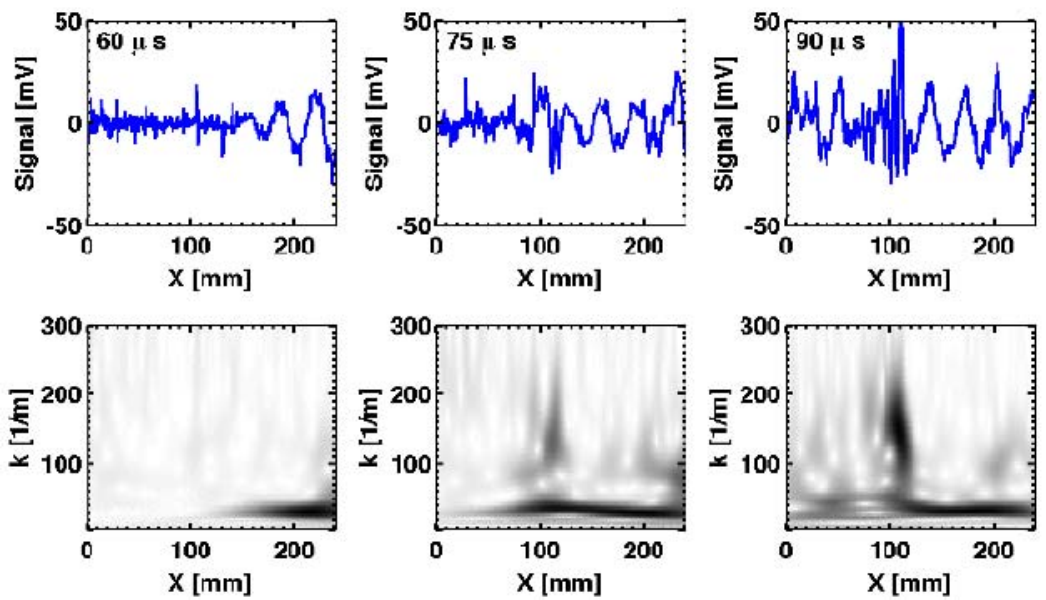

FIGURE 3. Recorded data and spatial-spectrogram representations for the composite stringer obtained at 60 (left column), 75 (center column), and $90 \mu$ s (right column). The incident, low wavenumber (approx. $30 \mathrm{~m}^{-1}$ ) wave propagates towards $\mathrm{x}=0$. High wavenumber (approx. $140 \mathrm{~m}^{-1}$ ) modes are created when the incident wave reaches the delaminations at $\mathrm{x}=120 \mathrm{~mm}$. As the wave continues to propagate, elevated wavenumber (approx. $45 \mathrm{~m}^{-1}$ ) is apparent from $\mathrm{x}=0$ to $\mathrm{x}=100 \mathrm{~mm}$.

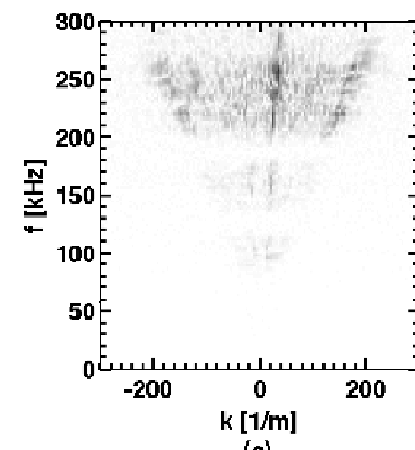

(a)

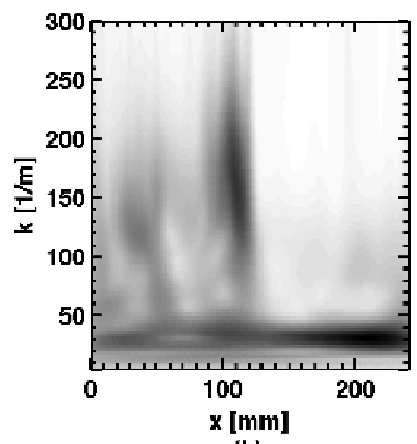

(b)

FIGURE 4. Two dimensional Fourier Transform (a) and spatial spectrogram (b) of composite stringer data presented in a normalized linear scale. The wavenumber bandwidths of the two transforms are comparable. 


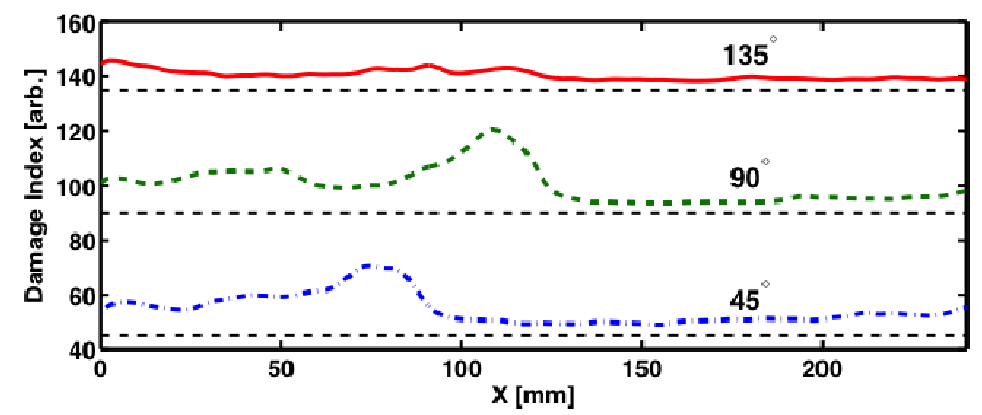

(a)

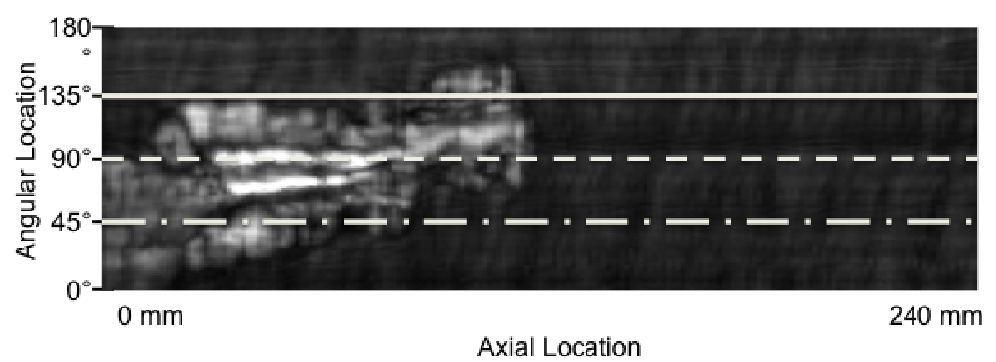

(b)

FIGURE 5. Computed damage indexes (a) corresponding to the three dashed lines in (b) and ultrasonic CScan (b) for the composite stringer. Offsets are added to the damage index equal to the angular location of the measurements for clarity.

Figure 5 shows a comparison of the C-scan obtained using an immersion scanning system with the wavenumber imaging results. The CWT coefficients for wavenumbers above $60 \mathrm{~m}^{-1}$ were summed for each location to obtain a damage index as shown in Figure 5a. The damage index at $45^{\circ}$ and $90^{\circ}$ agree with presence of the delamination in the c-scan. The damage index obtained at $135^{\circ}$ increases slightly compared to locations to the right but to a smaller extent than other inspections, showing less sensitivity to the presence of the delamination.

The utility of the technique for inspection of two-dimensional structures was investigated. First the 2D CWT is applied at a single wavenumber to determine if it is capable of acting as a filter to highlight and locate damage within a plate. The transform is performed at $\mathrm{k}=128 \mathrm{~m}^{-1}$ for the wavefield data at $50 \mu \mathrm{s}$ in order to highlight the A0 mode scattered by the incident S0 mode. Figure 6 shows the raw and filtered data for the four defects. The filtered data clearly shows the presence and approximate size of the defects and significantly reduces the amplitude of the S0 mode. Unsurprisingly, the deeper defects, $A$ and $C$, show a larger signal strength than the shallower defects with the same size, $B$ and D.

The filtered data gives a good indication of the presence and location of a defect but does not provide a measure of the depth of the defect. Previous research has shown that plate thinning will cause the wave speed of the A0 mode to decrease [13]. This is observed in the raw data by the distortion of the circular wavefronts. The extent of the thinning is indicated by the shift in wavenumber within the region of the defect. To measure the wavenumber, the spectrum is calculated at each location and the dominant wavenumber is determined by a centroid calculation. The resulting wavenumber maps are shown in Figures $7 \mathrm{c}$ and $7 \mathrm{~d}$. Edge effects are present at the boundaries of the scanned regions where the wavenumber is artificially increased. The wavenumber is also higher in the defect regions than in the pristine regions and a larger increase is present within defect $\mathrm{A}$ (from 97 to 105) than within defect B (from 97 to 100). The smaller defects did not show a clear 
difference between the two depths, possibly due to the defects being on the order of a wavelength in size.

\section{SUMMARY AND CONCLUSIONS}

The utility of continuous wavelet transform methods has been presented for visualizing and measuring the severity of defects in wavefield data. New methods were introduced for filtering wavefield data using both the one- and two-dimensional CWT. Data was processed that was obtained in linear paths along the length of a composite airframe stringer and in Cartesian grids in the vicinity of machined pockets in an aluminum plate.

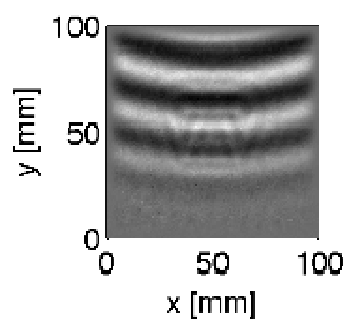

(a)

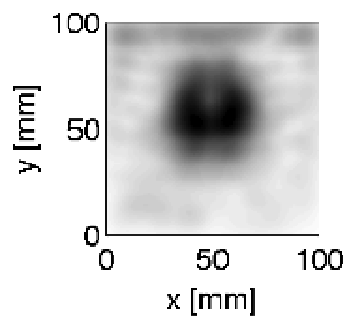

(e)

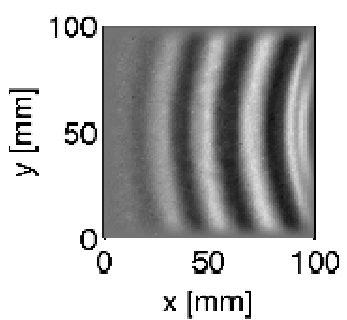

(b)

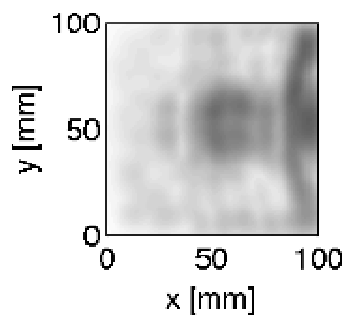

(f)

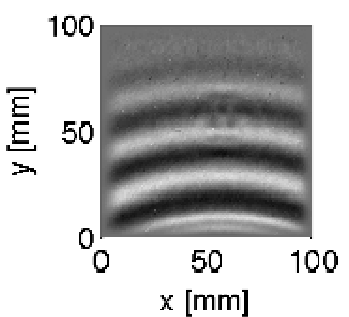

(c)

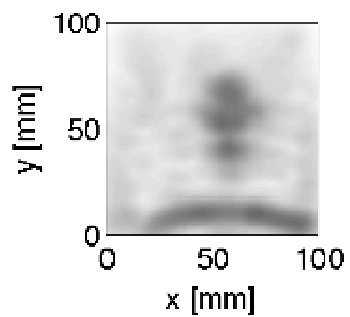

(g)

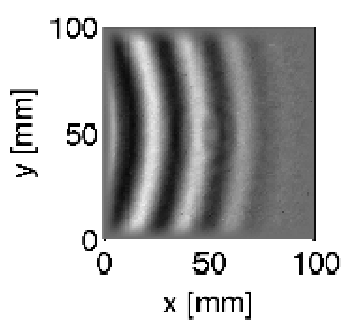

(d)

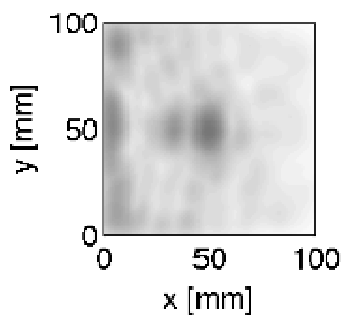

(h)

FIGURE 6. Raw and filtered data showing location and size of pockets machined in $2.2 \mathrm{~mm}$ thick aluminum plate. Recorded data obtained in the vicinity of defects A-D (a-d). Filtered data corresponding to (a-d) at $\mathrm{k}=128 \mathrm{~m}^{-1}(\mathrm{e}-\mathrm{h})$ where darker regions represents larger amplitude at the filtered wavenumber.

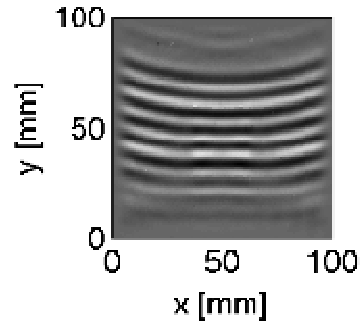

(a)

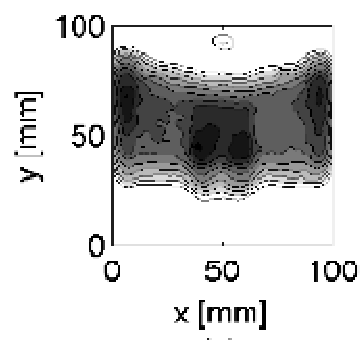

(c)

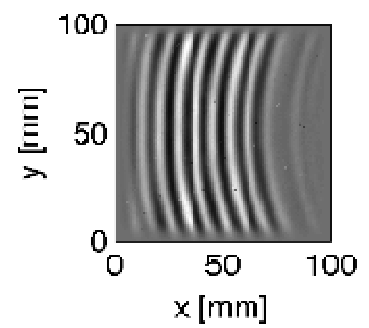

(b)

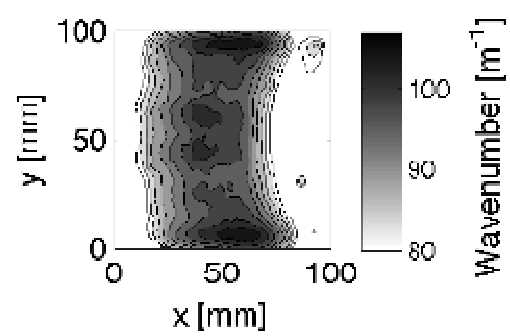

(d)

FIGURE 7. Recorded data and measured wavenumber obtained at $80 \mu$ s for Defect A (a) \& (c) and Defect B (b) \& (d). Wavenumber measurements show increased wavenumber within defects due to plate thinning. 
By applying the one dimensional CWT, the wavenumber spectrum of the acoustic wavefield is determined as a function of location along the scan line, permitting local propagation characteristics to be observed. The method allows visualization of wavenumber as the wave propagates through defect regions with significant changes in geometry. The presence of elevated wavenumber has been shown to correlate to overwrap delaminations in composite stringer structures. Thresholding the spectrum is a simple and effective means of providing an indicator of presence of damage.

The presented analysis of two dimensional wavefield data provides quantitative measurements of the wavenumber spectrum irrespective of propagation direction. This permits visualization of damage through simple filtering schemes or more advanced processing of the wavenumber spectrum to measure propagation characteristics.

\section{REFERENCES}

1. V. Giurgiutiu, A. Zagral, and J. J. Bao, "Piezoelectric Wafer Embedded Active Sensors for Aging Aircraft Structural Health Monitoring," Structural Health Monitoring, 1(1), pp. 41-61 (2002).

2. A.J. Croxford, P. D. Wilcox, B. W. Drinkwater, and G. Konstantinidis, Proc. R. Soc. A 8, 463(2087), pp. 2961-2981 (2007).

3. T. E. Michaels, M. Ruzzene, and J. E. Michaels, "Frequency-wavenumber domain methods for analysis of incident and scattered guided wave fields," Health Monitoring of Structural and Biological Systems, 7295, T. Kundu (Ed.), SPIE, pp. 729513-1-12, 2009

4. M. Ruzzene, S. M. Jeong, T. E. Michaels, J. E. Michaels and B. Mi, "Simulation and measurement of ultrasonic waves in elastic plates using laser vibrometry," Review of Quantitative Nondestructive Evaluation 760, D. O. Thompson and D. E. Chimenti (Eds.), AIP, pp. 172-9, 2005

5. T. E. Michaels, M. Ruzzene, and J. E. Michaels, "Incident Wave Removal Through Frequency-Wavenumber Filtering of Full Wavefield Data," Review of Progress in Quantitative Nondestructive Evaluation, 1096, D. O. Thompson and D. E. Chimenti (Eds.), AIP, pp. 172-179, 2008.

6. T. E. Michaels, M. Ruzzene, and J. E. Michaels, "Frequency-wavenumber domain analysis of guided wavefields," Ultrasonics 51, pp. 452-466 (2011).

7. V. K. Sharma, S. Hanagud and M. Ruzzene, AIAA Journal, 44(4), pp. 919-922, 2006.

8. W. J. Staszewski, B. C. Lee, L. Mallet and F. Scarpa, Smart Materials and Structures, 13, pp. 251-260 (2004).

9. D. Cerniglia, B. B. Djordjevic, "Analysis of Laser-Generated Lamb Waves with Wavelet Transform," Nondestructuve Characterization of Materials 11, Green, Robert E., Djordjevic, B. Boro and Hentschel, Manfred P. (Eds.), Springer, pp. 63-7, 2003.

10. S. Mallat, Wavelet Tour of Signal Processing, 3rd ed., Academic Press, Burlington, MA, 2008, p. 17.

11. C. Leckey, M. Rogge, C. Miller, M. Hinders, "Multiple-mode Lamb Wave Scattering Simulations using 3D Elastodynamic Finite Integration Technique," Ultrasonics, In Press, DOI: 10.1016/j.ultas.2011.08.003.

12. P. Marical, M. Ech-Cherif El-Kettani, M.V. Predoi, "Guided waves in elastic plates with Gaussian section variation: Experimental and numerical results," Ultrasonics, 47, pp. 1-9 (2007).

13. J. Pei, M. Yousuf, F. Degertekin, B. Honein, B. Khuri-Yakub, "Lamb wave tomography and its application in pipe erosion/corrosion monitoring," Research in Nondestructuve Evaluation, 8(4), pp. 189-197 (1996). 